\title{
Glycosylation using Unprotected Alkynyl Donors
}

\author{
Sreeman K. Mamidyala* and M. G. Finn* \\ Department of Chemistry and The Skaggs Institute of Chemical Biology, \\ 10550 North Torrey Pines Road, The Scripps Research Institute, \\ La Jolla CA 92037 \\ e-mail: sreeman@scripps.edu,mgfinn@scripps.edu \\ Supporting Information
}

\section{Contents}

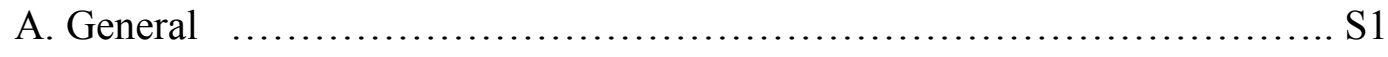
B. Synthesis of 2-butynyl donors 27-29. ................................. S2
C. ${ }^{1} \mathrm{H}$ NMR spectra of glycosyl donors and products ......................... S3
D. Mass spectral data of the glycosylated compounds........................ S19

A. General. Solvents THF, acetonitrile, diethyl ether, dichloromethane, and toluene were dried by passage through activated alumina columns. ${ }^{1}$ Chromatography was performed using SINGLE StEP pre-packed MPLC columns. Reaction progress was monitored by TLC using silica gel 60 F-254 with uv detection. Silica gel $60(40-63 \mu \mathrm{m})$ was used for column chromatography. Solutions of phosphomolybdic acid or anisaldehyde/EtOH were used in addition to UV light with fluorescent TLC plates. Reactions requiring anhydrous conditions were performed under nitrogen. ${ }^{1} \mathrm{H}$ and ${ }^{13} \mathrm{C}$ NMR spectra were recorded on a Varian 300 spectrometer in $\mathrm{CDCl}_{3}$, $\mathrm{CD}_{3} \mathrm{OD}$, or DMSO- $d_{6}$ solvent. NMR data are presented as follows: chemical shift (ppm), multiplicity $(s=$ singlet, $d=$ doublet, $t=$ triplet, $q=$ quartet, quin = quintet, sep = septet, $m=$ multiplet, $b=$ broad), coupling constant $(\mathrm{Hz})$ and integration. Routine mass spectra (LCMS) were obtained using an Agilent 1100 (ESI MSD) with mobile phase composed of 9:1 $\mathrm{CH}_{3} \mathrm{CN}: \mathrm{H}_{2} \mathrm{O}$ containing $0.1 \% \mathrm{CF}_{3} \mathrm{CO}_{2} \mathrm{H}$.

${ }^{1}$ (a) Pangborn, A. B.; Giardello, M. A.; Grubbs, R. H.; Rosen, R. K.; Timmers, F. J. Organometallics 1996, 15, 1518-1520. (b) Alaimo, P. J.; Peters, D. W.; Arnold, J.; Bergman, R. G. J. Chem. Ed. 2001, 78, 64 


\section{B. Synthesis of 2-Butynyl Donors 27-29.}

Figure S1. Synthesis of 2-galactosyl donors.
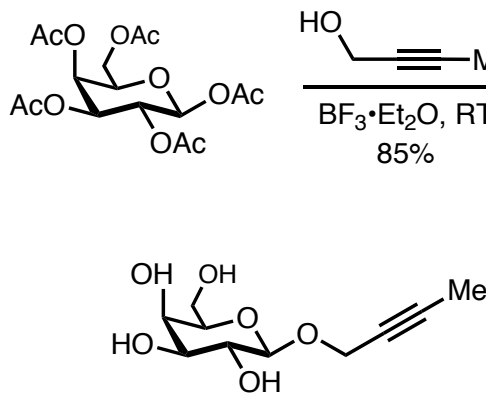

28

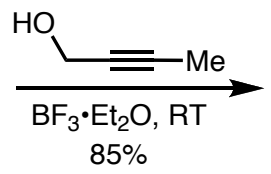

$85 \%$

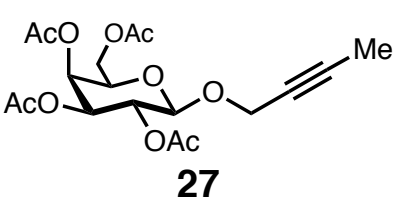

$\underset{\mathrm{RT}, 12 \mathrm{~h}}{\mathrm{NaOMe}, \mathrm{MeOH}}$

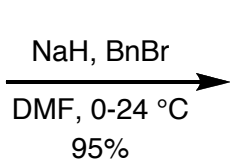

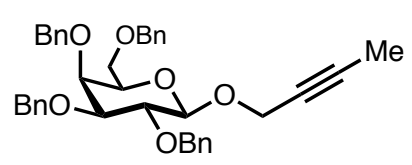

29

2-Butynyl-2,3,4,6-tetra- $O$-acetyl- $\beta$-D-galactopyranoside (27)

To a solution of penta- $O$-acteyl-D-galatcose $(4.0 \mathrm{~g}, 10.2 \mathrm{mmol})$ in dichloromethane $(80.0$ $\mathrm{mL}$ ) was added 2-butynyl-1-ol $(1.54 \mathrm{~mL}, 20.4 \mathrm{mmol})$. The reaction was cooled to $0{ }^{\circ} \mathrm{C}$ and $\mathrm{BF}_{3} . \mathrm{Et}_{2} \mathrm{O}(2.5 \mathrm{~mL}, 20.4 \mathrm{mmol})$ was added slowly. The mixture was allowed to warm to room temperature with stirring, while monitoring the reaction progress by TLC. Upon completion ( $5 \mathrm{~h})$ the reaction was diluted with dichloromethane and neutralized with aq. $\mathrm{K}_{2} \mathrm{CO}_{3}$. The organic layer was washed with water, separated and dried $\left(\mathrm{Na}_{2} \mathrm{SO}_{4}\right)$. The solvent was removed and the crude product was purified by flash column chromatography to give $\mathbf{2 7}$ as a hygroscopic white solid (3.4 g, 85\%). Mp. $56{ }^{\circ} \mathrm{C} .{ }^{1} \mathrm{H}$ NMR $\left(300 \mathrm{MHz}, \mathrm{CDCl}_{3}\right): \delta 1.83$ (t, $\left.3 \mathrm{H}, J=3.0 \mathrm{~Hz}\right), 1.95$ (s, 3H), $2.01(\mathrm{~s}, 3 \mathrm{H}), 2.03(\mathrm{~s}, 3 \mathrm{H}), 2.11(\mathrm{~s}, 3 \mathrm{H}), 3.84-3.94(\mathrm{~m}, 1 \mathrm{H}), 4.02-4.20(\mathrm{~m}, 2 \mathrm{H}), 4.30(\mathrm{q}, 2 \mathrm{H}, J=$ $3.0 \mathrm{~Hz}), 4.68(\mathrm{~d}, 1 \mathrm{H}, J=9.0 \mathrm{~Hz}), 5.02(\mathrm{dd}, 1 \mathrm{H}, J=3.0,12.0 \mathrm{~Hz}), 5.17(\mathrm{dd}, 1 \mathrm{H}, J=9.0,12.0 \mathrm{~Hz})$, $5.36(\mathrm{~d}, 1 \mathrm{H}, J=3.0 \mathrm{~Hz}) .{ }^{13} \mathrm{C} \mathrm{NMR}(75 \mathrm{MHz}, \mathrm{CDCl}): \delta 16.9\left(\mathrm{CH}_{3}\right), 17.0\left(\mathrm{CH}_{3}\right), 17.1\left(\mathrm{CH}_{3}\right), 17.2$ $\left(\mathrm{CH}_{3}\right), 53.0\left(\mathrm{CH}_{3}\right), 57.5(\mathrm{C}-3), 63.4(\mathrm{C}-2), 64.9\left(\mathrm{C}-6\right.$ ') $67.0\left(\mathrm{C}-5^{\prime}\right), 67.3(\mathrm{C}-3$ ' $), 70.0(\mathrm{C}-4$ '), 79.8 (C-2'), $94.9\left(\mathrm{C}^{\prime} 1^{\prime}\right), 165.9(\mathrm{C}=\mathrm{O}), 166.5(\mathrm{C}=\mathrm{O}), 166.6(\mathrm{C}=\mathrm{O}), 166.7(\mathrm{C}=\mathrm{O})$. Anal. (after drying under vacuum). Calcd for $\mathrm{C}_{18} \mathrm{H}_{24} \mathrm{O}_{10}: \mathrm{C}, 54.00 ; \mathrm{H}, 6.04 ; \mathrm{N}, 0.00$. Found: $\mathrm{C}, 54.13 ; \mathrm{H}$, $5.99 ; \mathrm{N}, 0.00$.

\section{2-Butynyl- $\beta$-D-galactopyranoside (28)}

To a solution of $27(2.4 \mathrm{~g}, 6.0 \mathrm{mmol})$ in methanol $(15 \mathrm{~mL})$ was added $2 \mathrm{~N} \mathrm{NaOMe}(1.0$ $\mathrm{mL})$. The reaction was stirred at room temperature, monitoring by TLC. Upon completion $(5 \mathrm{~h})$ the mixture was neutralized with Amberlite IR $120(\mathrm{H}+)$ resin. The resin was filtered and solvent was evaporated to give 28 as a hygroscopic white solid (1.25 g, 90\%). Mp. $154{ }^{\circ} \mathrm{C} .{ }^{1} \mathrm{H}$ NMR $\left(300 \mathrm{MHz}, \mathrm{CDCl}_{3}\right): \delta 1.83(\mathrm{t}, 3 \mathrm{H}, J=3.0,6.0 \mathrm{~Hz}), 3.45-3.60(\mathrm{~m}, 3 \mathrm{H}), 3.72-3.82(\mathrm{~m}, 2 \mathrm{H}), 4.30$ $(2 \mathrm{~d}, 1 \mathrm{H}, J=3.0 \mathrm{~Hz}), 4.35-4.40(\mathrm{~m}, 2 \mathrm{H}) 4.42(\mathrm{~d}, 1 \mathrm{H}, J=9.0 \mathrm{~Hz}) .{ }^{13} \mathrm{C}$ NMR $\left(75 \mathrm{MHz}, \mathrm{CDCl}_{3}\right): \delta$ $53.9\left(\mathrm{CH}_{3}\right), 59.3$ (C-3), 67.1 (C-2), 69.1 (C-6'), 71.7 (C-5'), 72.3 (C-3'), 73.5 (C-4'), 80.2 (C2'), 99.4 (C-1'). Anal. (after drying under vacuum). Calcd for $\mathrm{C}_{10} \mathrm{H}_{16} \mathrm{O}_{6}$ : C, 51.72; H, 6.95; N, 0.00. Found: C, 51.82; H, 6.84; N, 0.00 . 


\section{2-Butynyl-2,3,4,6-tetra-O-benzyl- $\beta$-D-galactopyranoside (29)}

The solution of $28(0.4 \mathrm{~g}, 1.7 \mathrm{mmol})$ in DMF $(3.0 \mathrm{~mL})$ was cooled to $0{ }^{\circ} \mathrm{C}$ and added $\mathrm{NaH}(0.24 \mathrm{~g}, 10.2 \mathrm{mmol})$. The reaction was stirred at room temperature for $1 \mathrm{~h}$. The reaction was monitored by TLC and when completed it was neutralized with ice cold water. The reaction mxture was extracted with diethyl ether $(40.0 \mathrm{~mL})$, washed with water. The organic layer was separated, dried $\left(\mathrm{Na}_{2} \mathrm{SO}_{4}\right)$ and evaporated to give the title compound as a hygroscopic white solid (0.8 g, 80\%). Mp. $45^{\circ} \mathrm{C} .{ }^{1} \mathrm{H}$ NMR $\left(300 \mathrm{MHz}, \mathrm{CDCl}_{3}\right): \delta 1.82(\mathrm{t}, 3 \mathrm{H}, J=3.0 \mathrm{~Hz}), 3.50-3.60$ $(\mathrm{m}, 4 \mathrm{H}), 3.84(\mathrm{dd}, 1 \mathrm{H}, J=6.0,9.0 \mathrm{~Hz}), 3.88(\mathrm{~d}, 1 \mathrm{H}, J=3.0 \mathrm{~Hz}), 4.32-4.38(\mathrm{~m}, 2 \mathrm{H}), 4.40(\mathrm{~d}, 2 \mathrm{H}$, $J=6.0 \mathrm{~Hz}), 4.58(\mathrm{~s}, 1 \mathrm{H}), 4.62(\mathrm{~d}, 1 \mathrm{H}, J=9.0 \mathrm{~Hz}), 4.64-4.80(\mathrm{~m}, 3 \mathrm{H}), 4.90-5.00(\mathrm{~m}, 2 \mathrm{H}), 7.02-$ $7.60(\mathrm{~m}, 20 \mathrm{H}) .{ }^{13} \mathrm{C}$ NMR $(75 \mathrm{MHz}, \mathrm{CDCl} 3): \delta 52.6\left(\mathrm{CH}_{3}\right), 65.0(\mathrm{C}-3), 69.4\left(\mathrm{CH}_{2}\right), 69.6\left(\mathrm{CH}_{2}\right)$, $69.7\left(\mathrm{CH}_{2}\right), 69.8\left(\mathrm{CH}_{2}\right), 70.7$ (C-6'), 70.9 (C-5'), 71.3 (C-4'), 75.6 (C-3'), 78.5 (C-2), 78.9 (C2'), 97.7 (C-1'), 120-135 (Ar-C). Anal. (after drying under vacuum). Calcd for $\mathrm{C}_{38} \mathrm{H}_{40} \mathrm{O}_{6}$ : C, 77.00; H, 6.80; N, 0.00. Found: C, 76.81; H, 6.83; N, 0.00 .

\section{C. ${ }^{1}$ H NMR Spectra of Glycosyl Donors and Products}

All compounds reported here have been previously described, with characterization data reported in the papers cited in Table 2 of the main text. ${ }^{1} \mathrm{H}$ NMR spectra (shown here) were obtained to verify the identities of the compounds by comparison to published data.

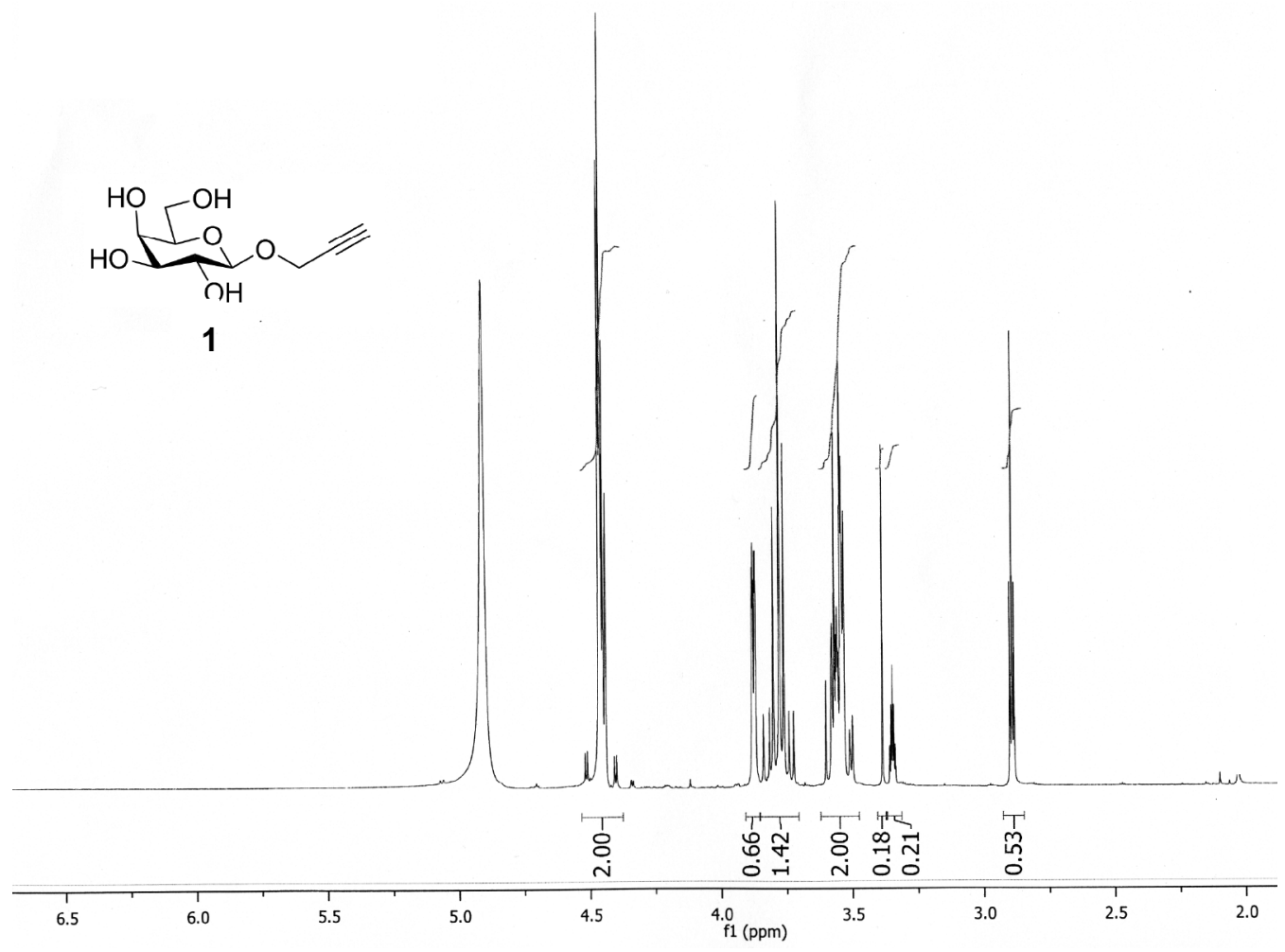




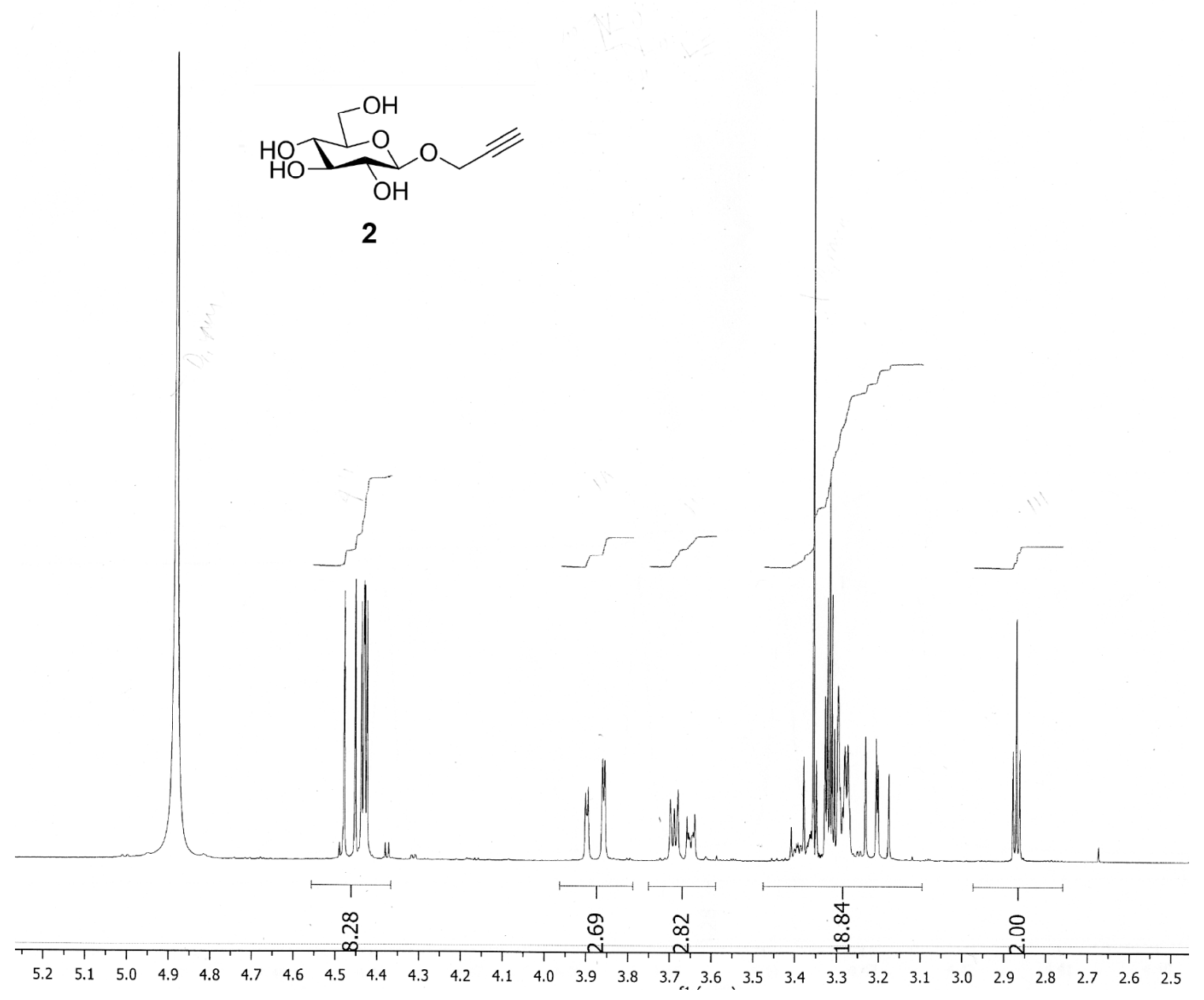



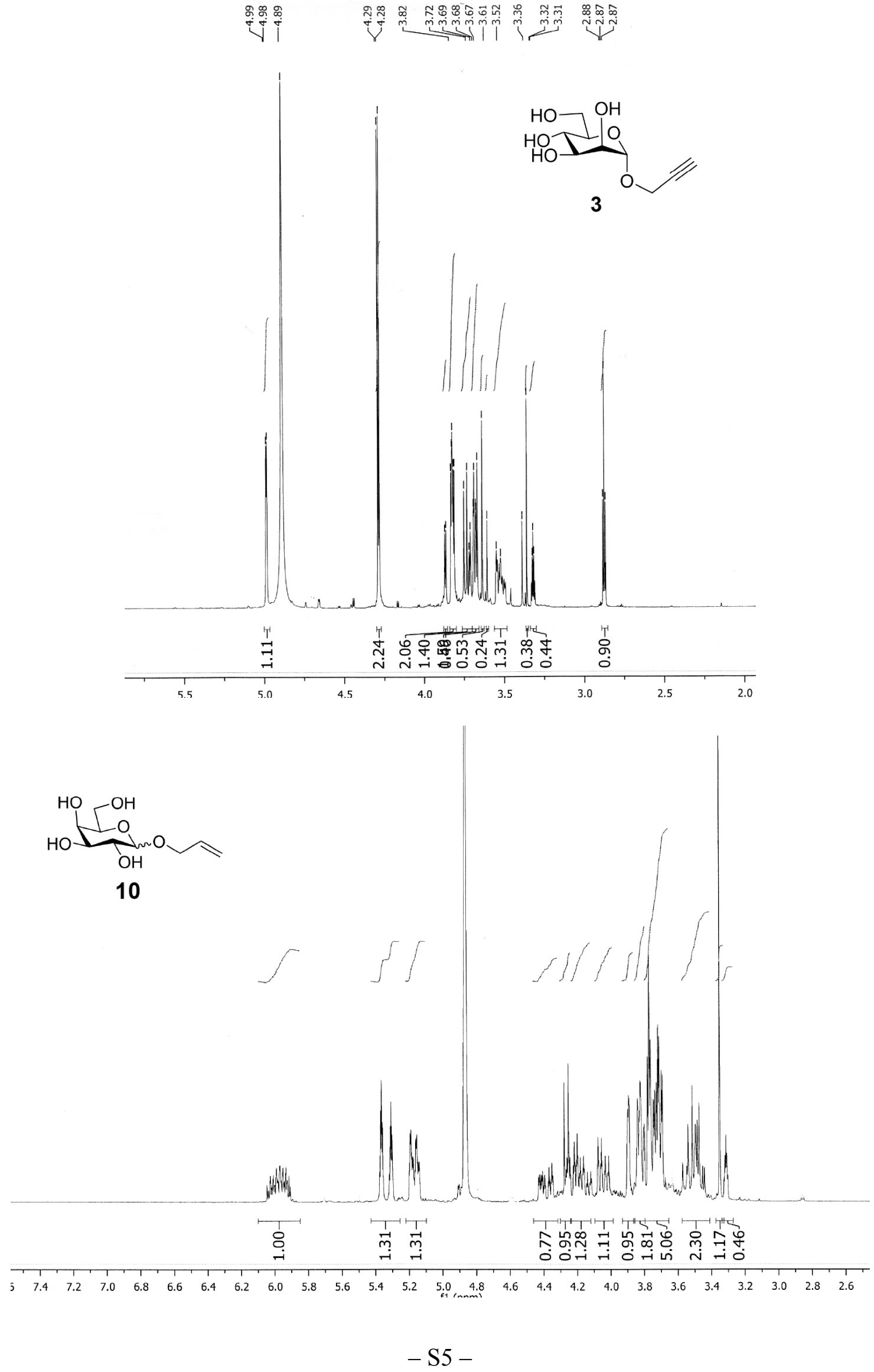

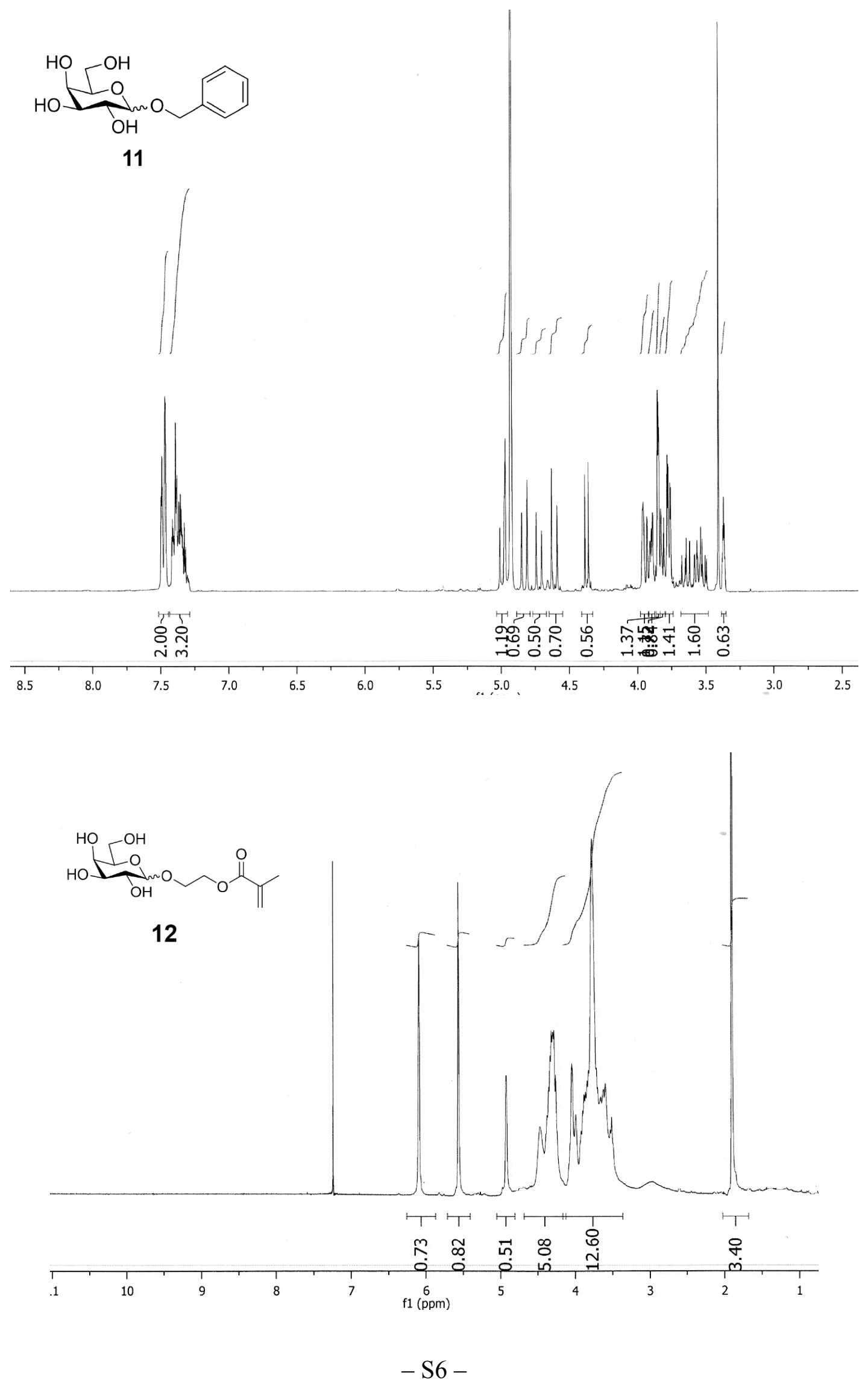

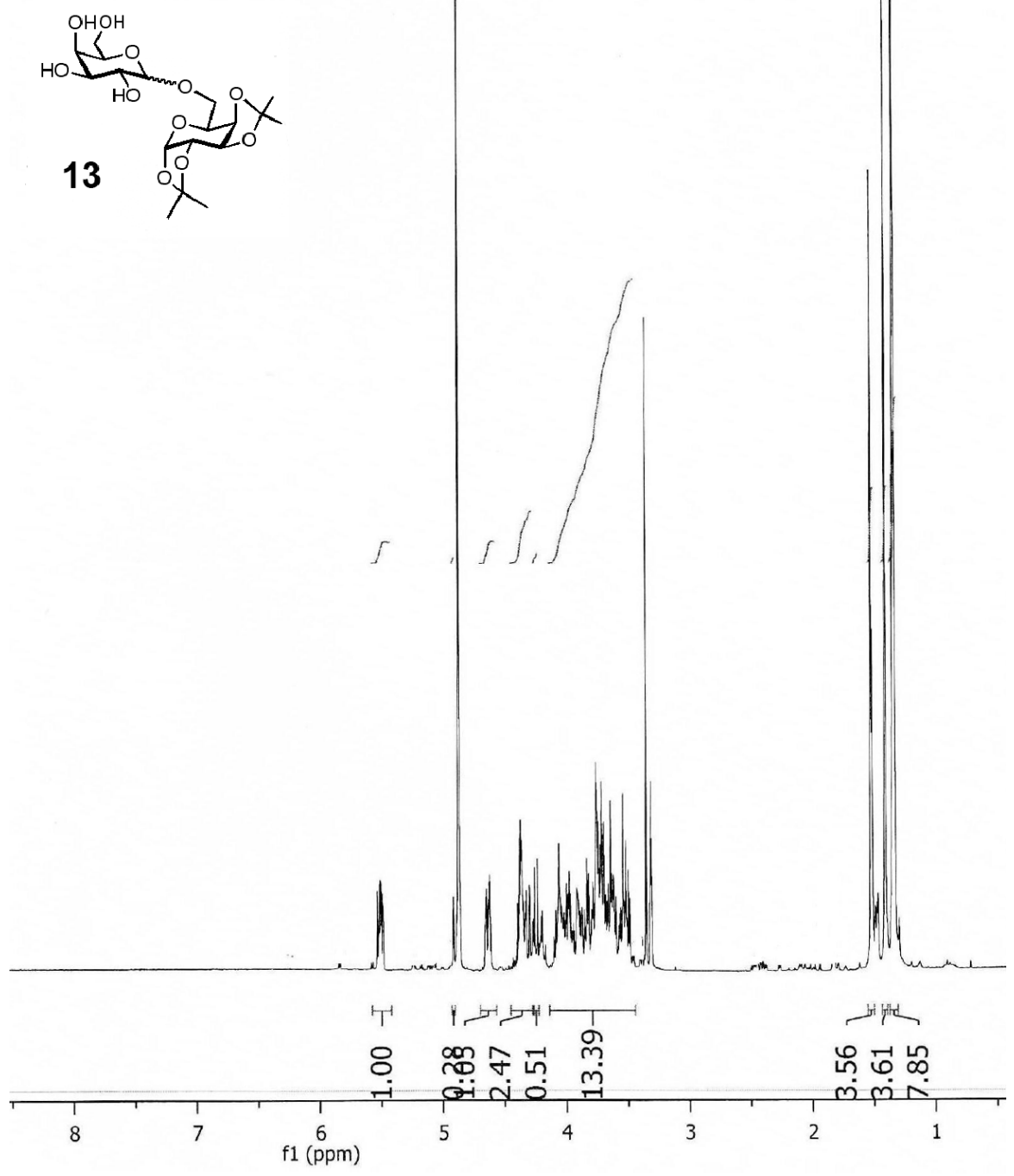


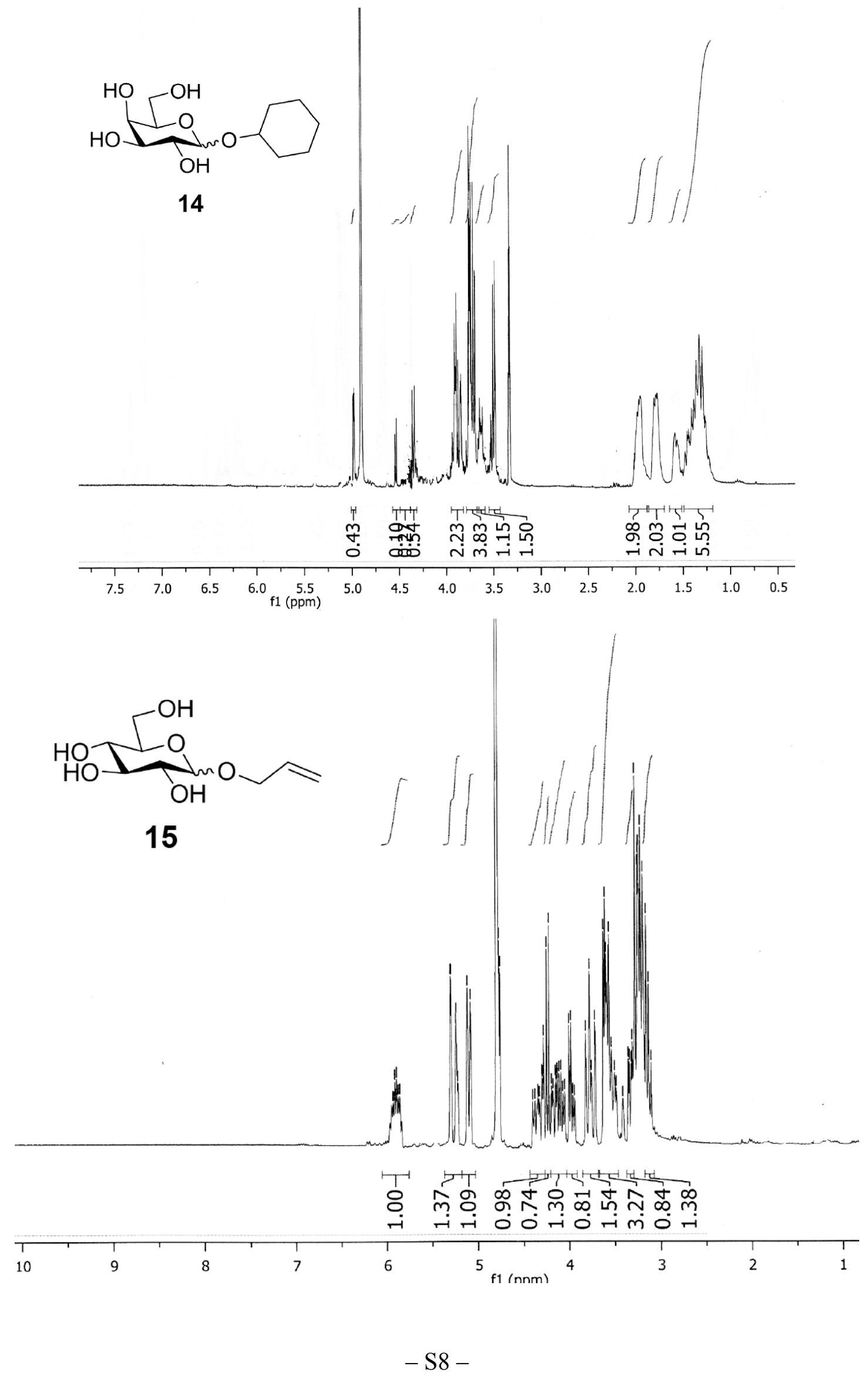



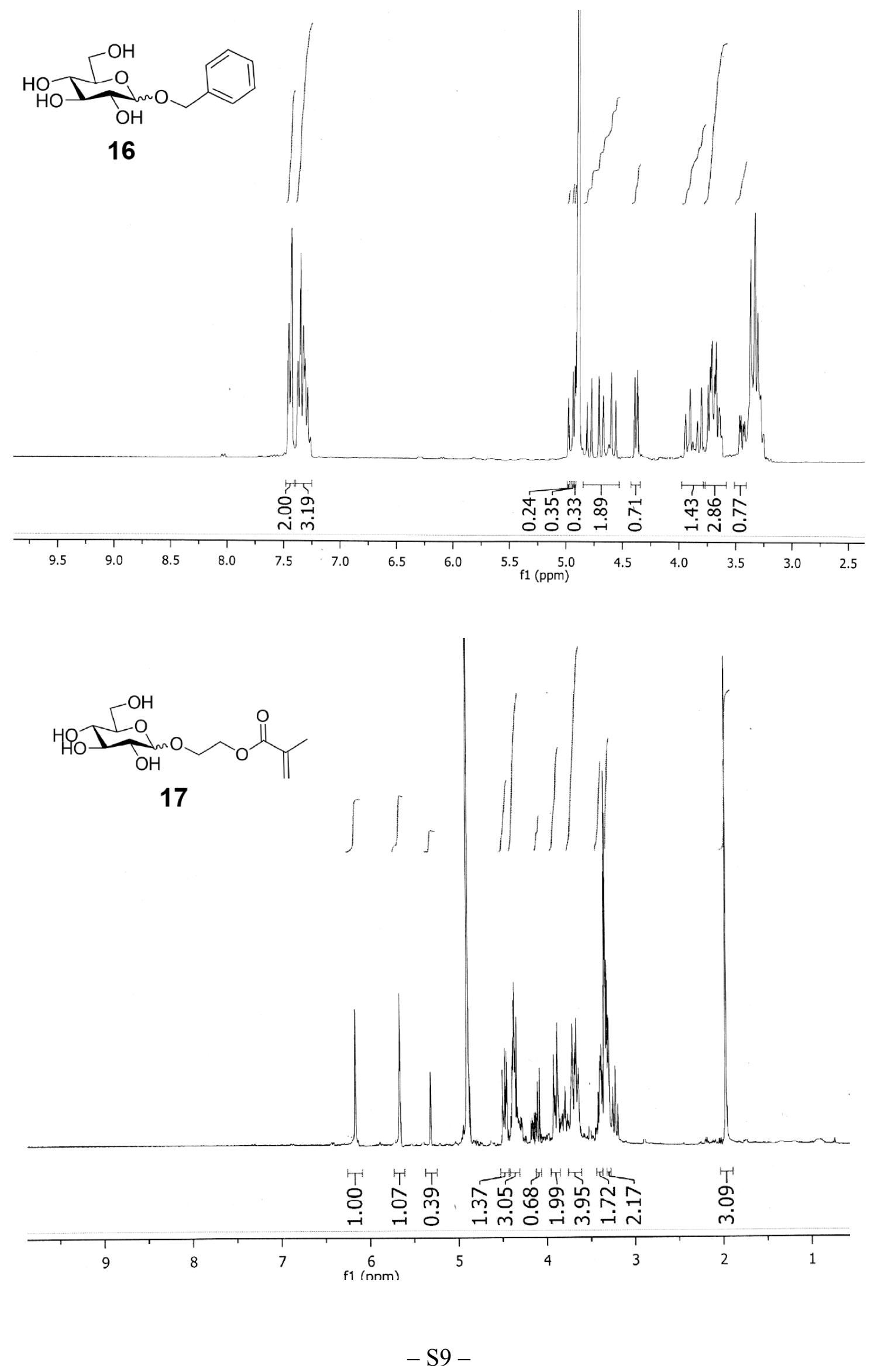


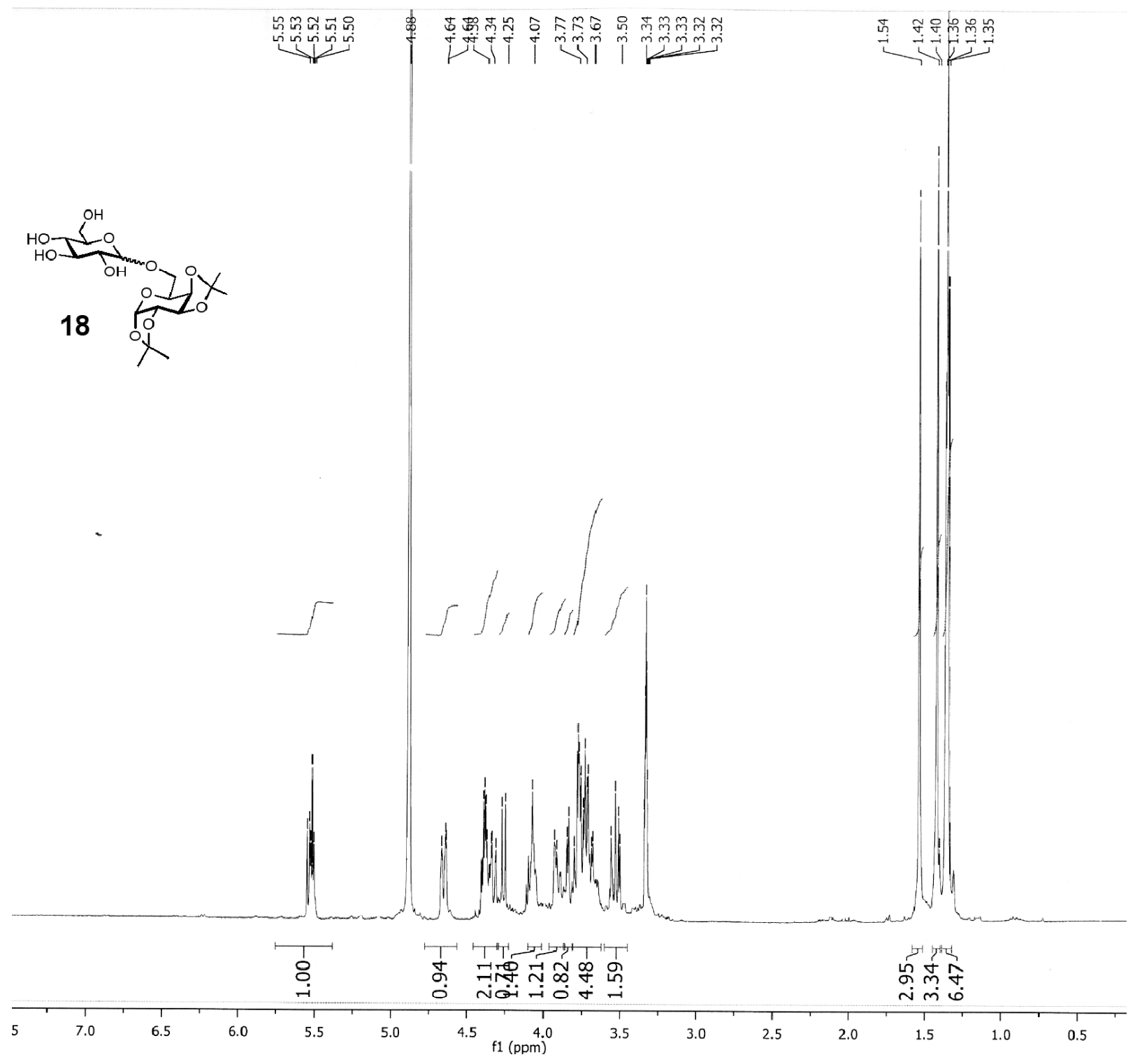




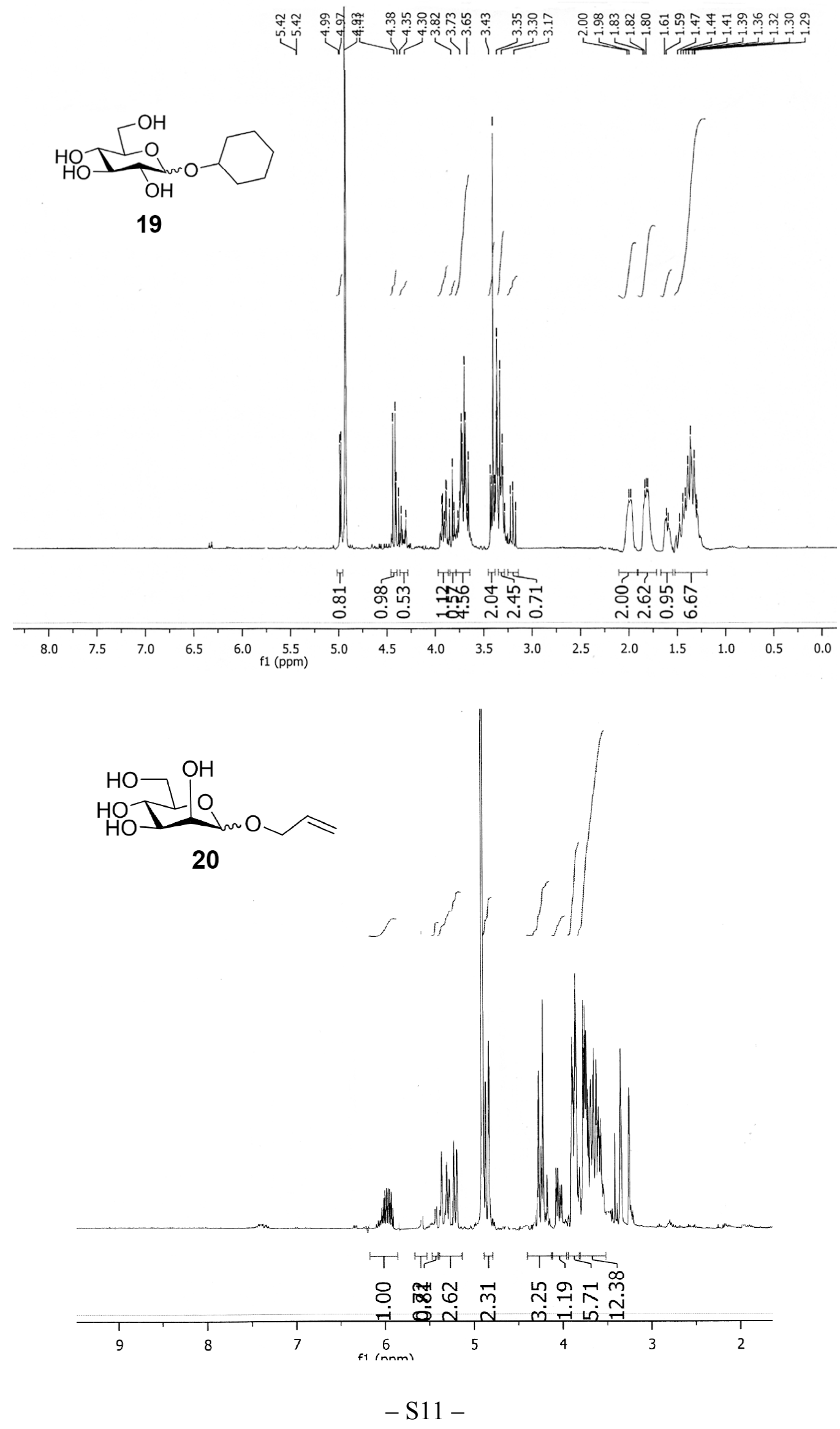



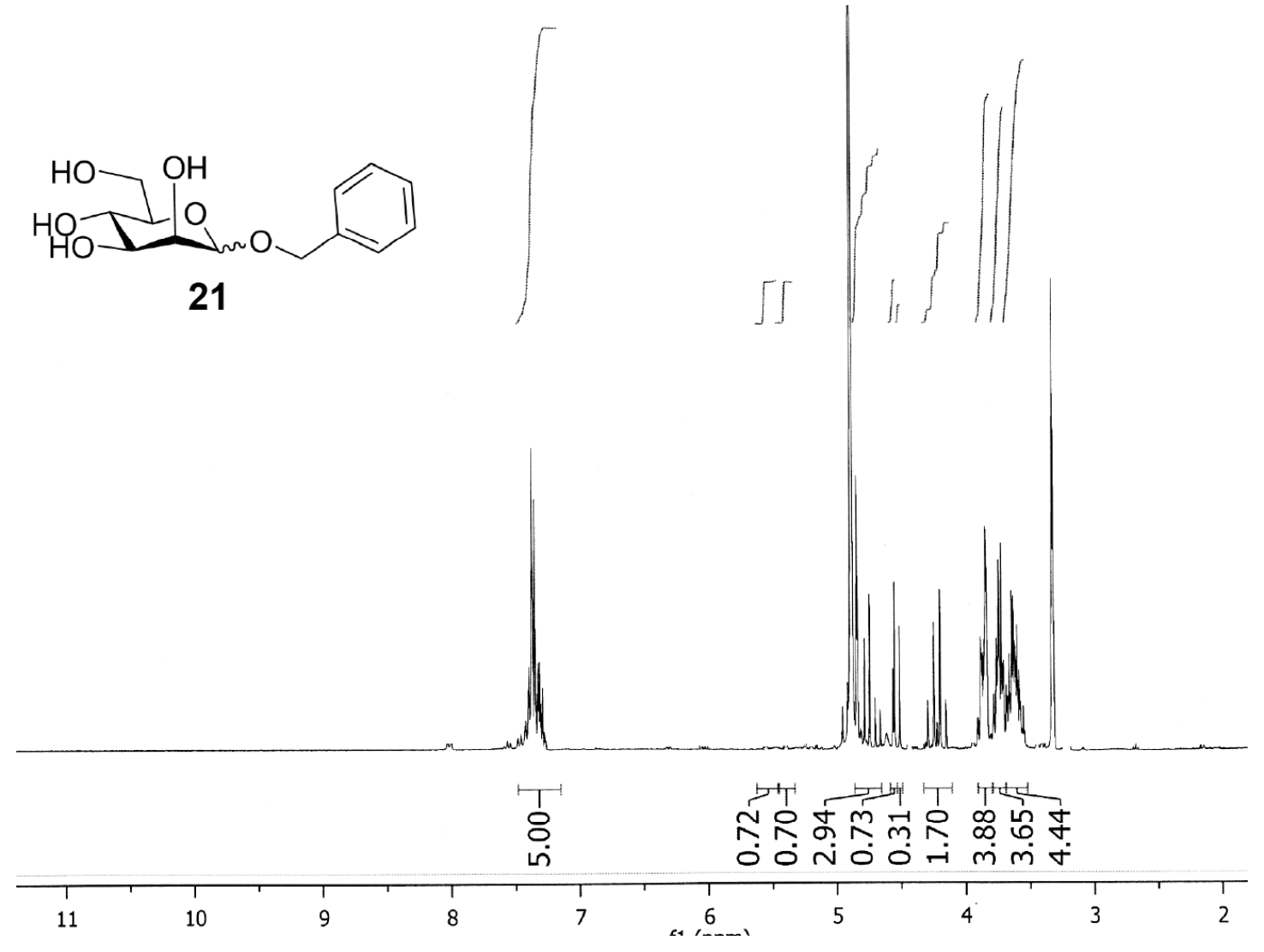


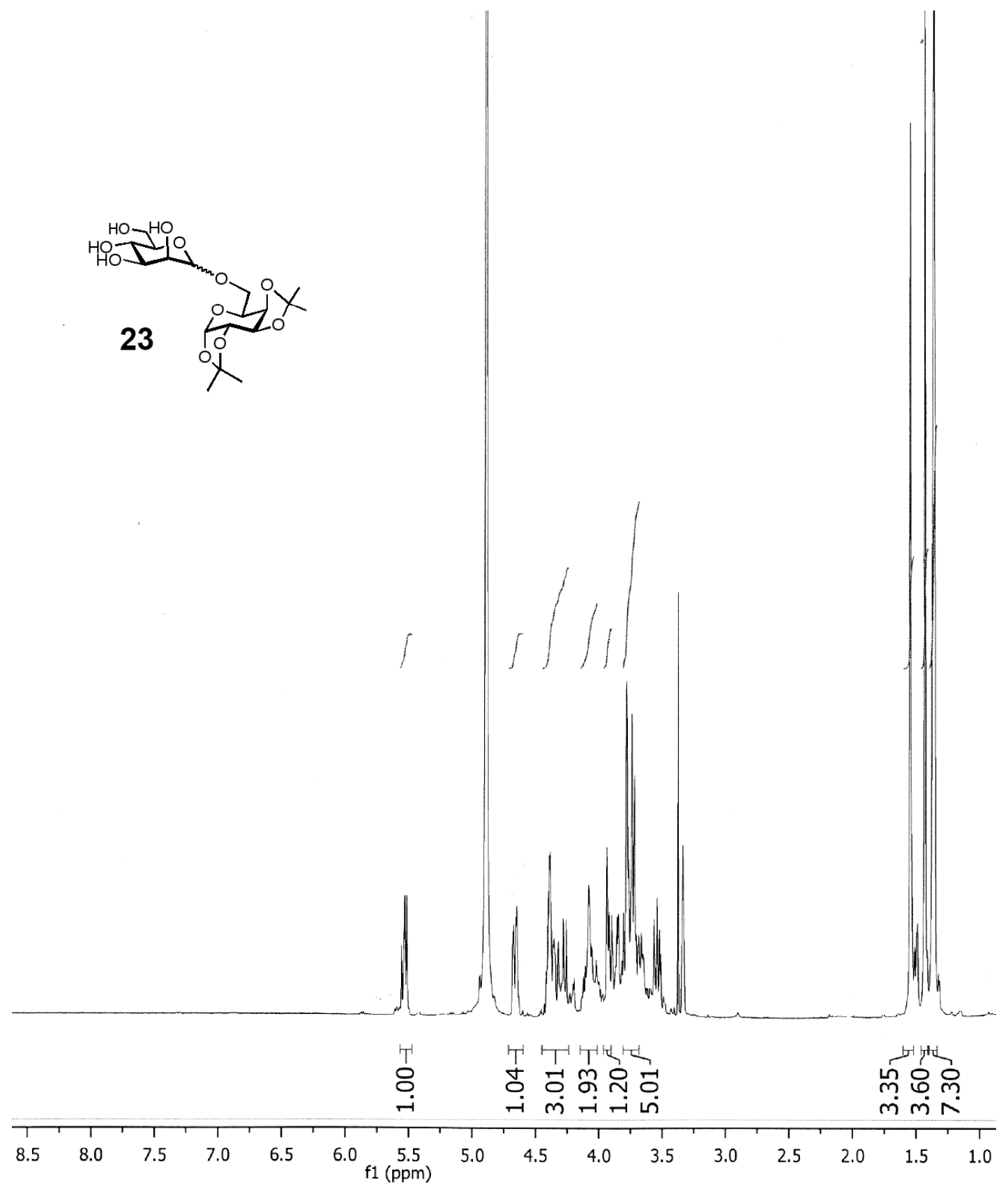



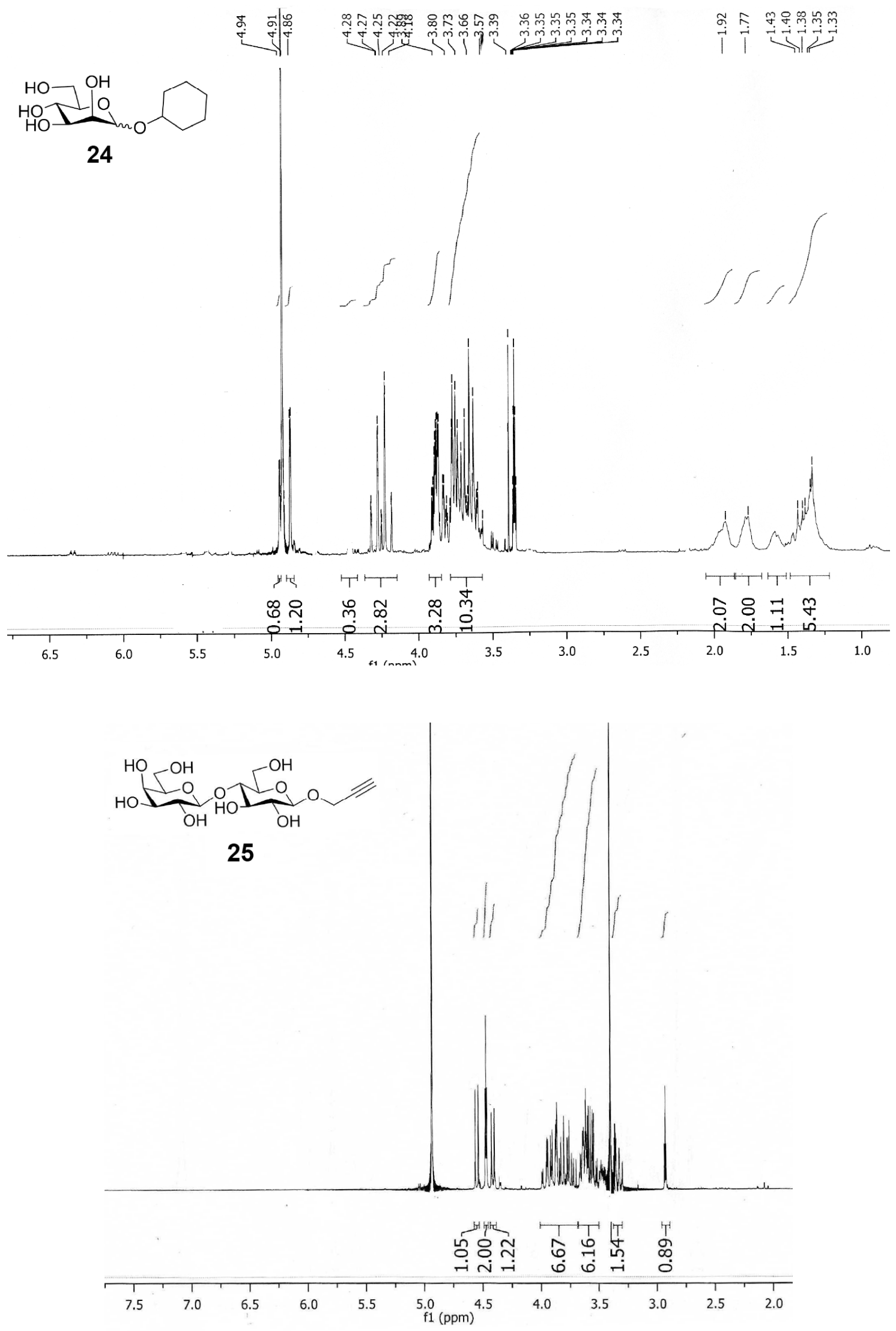


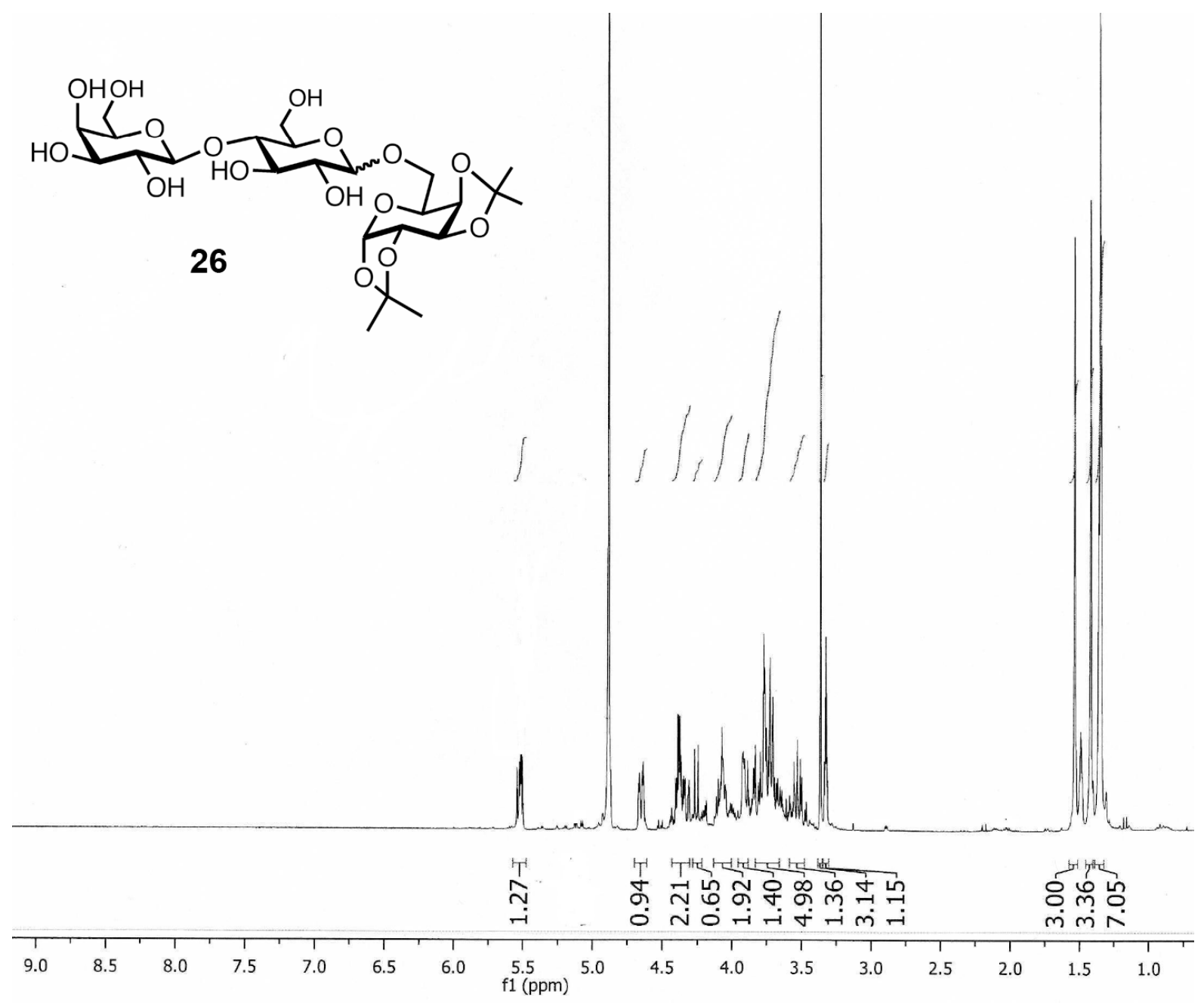





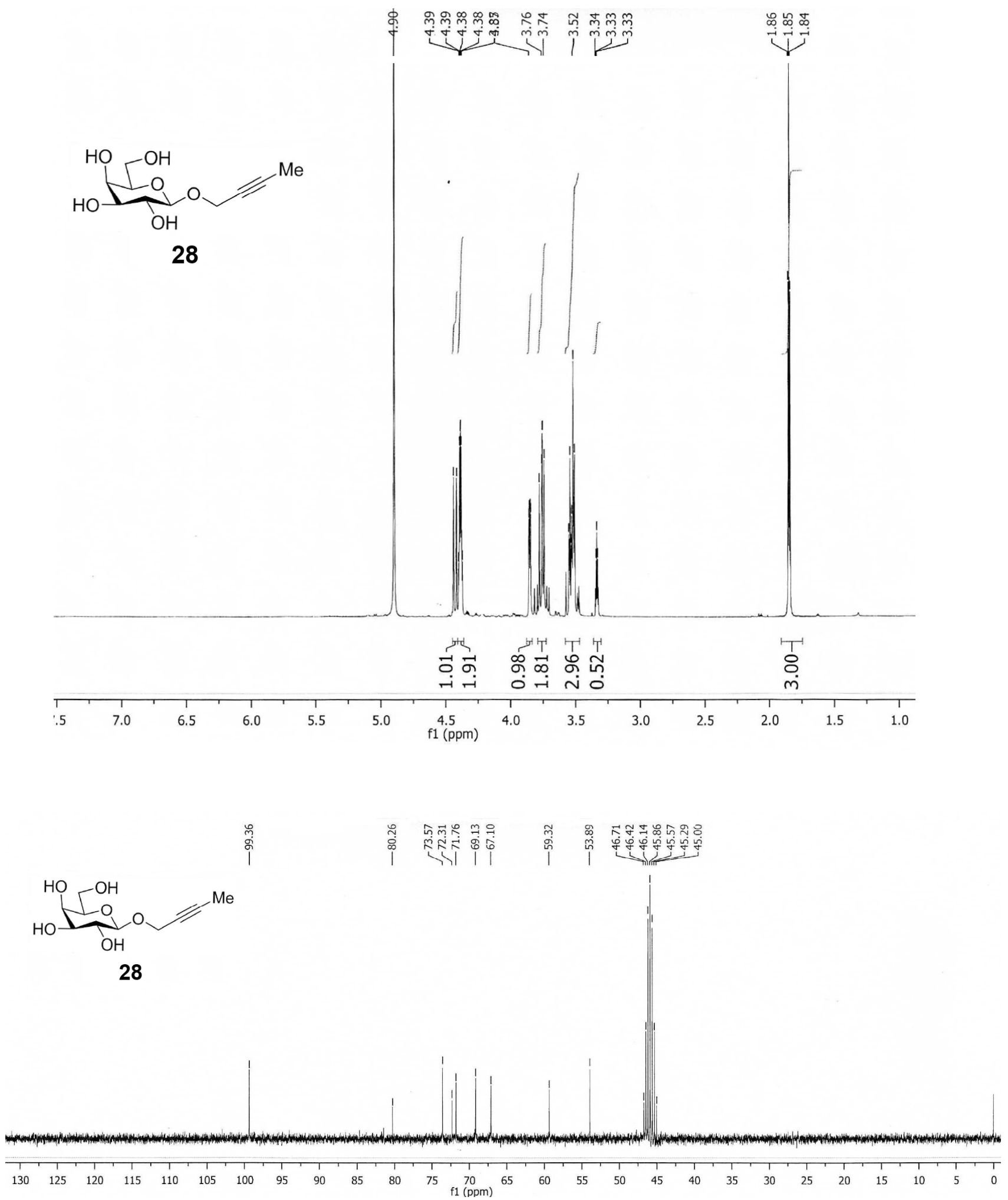

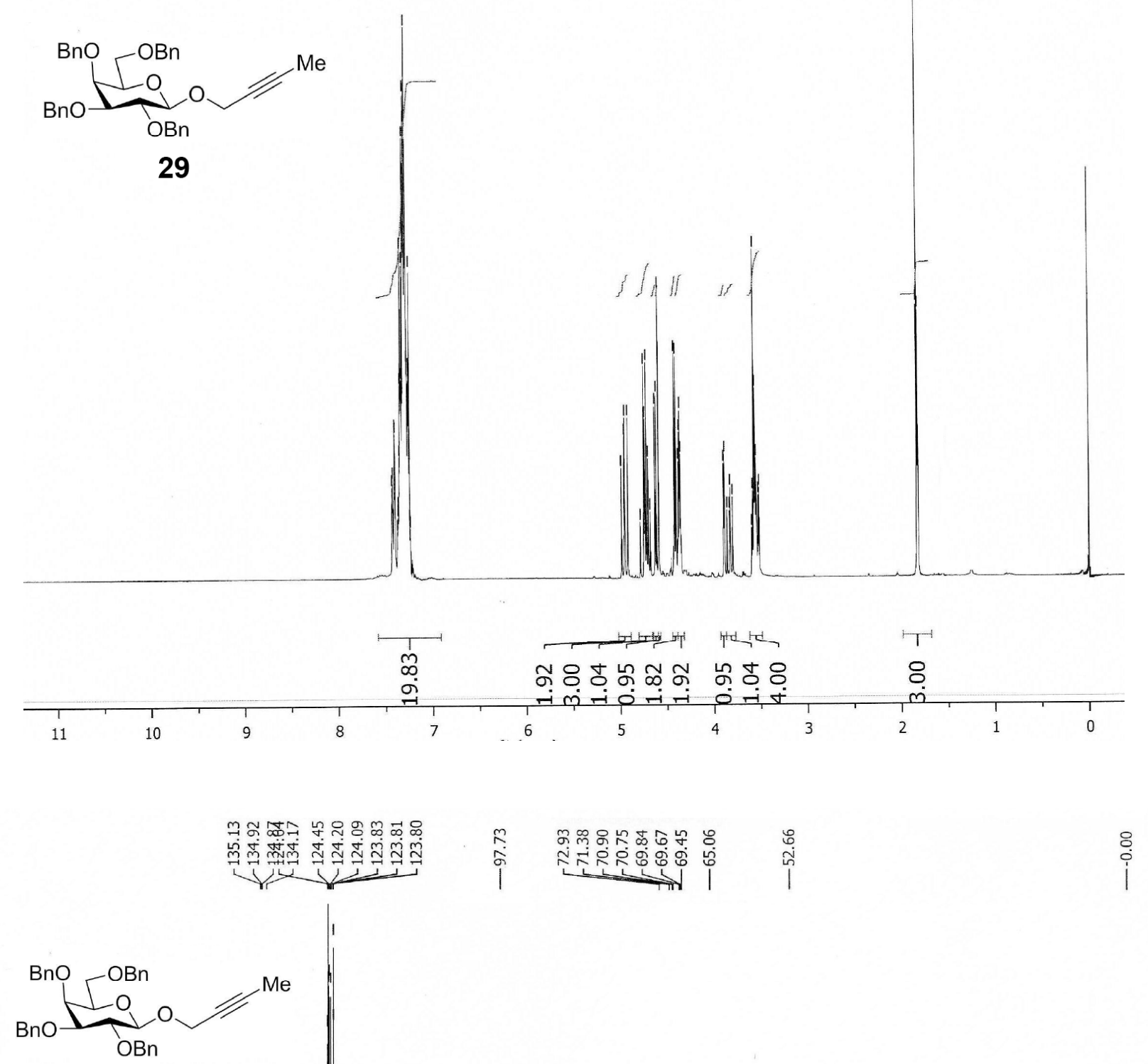

29

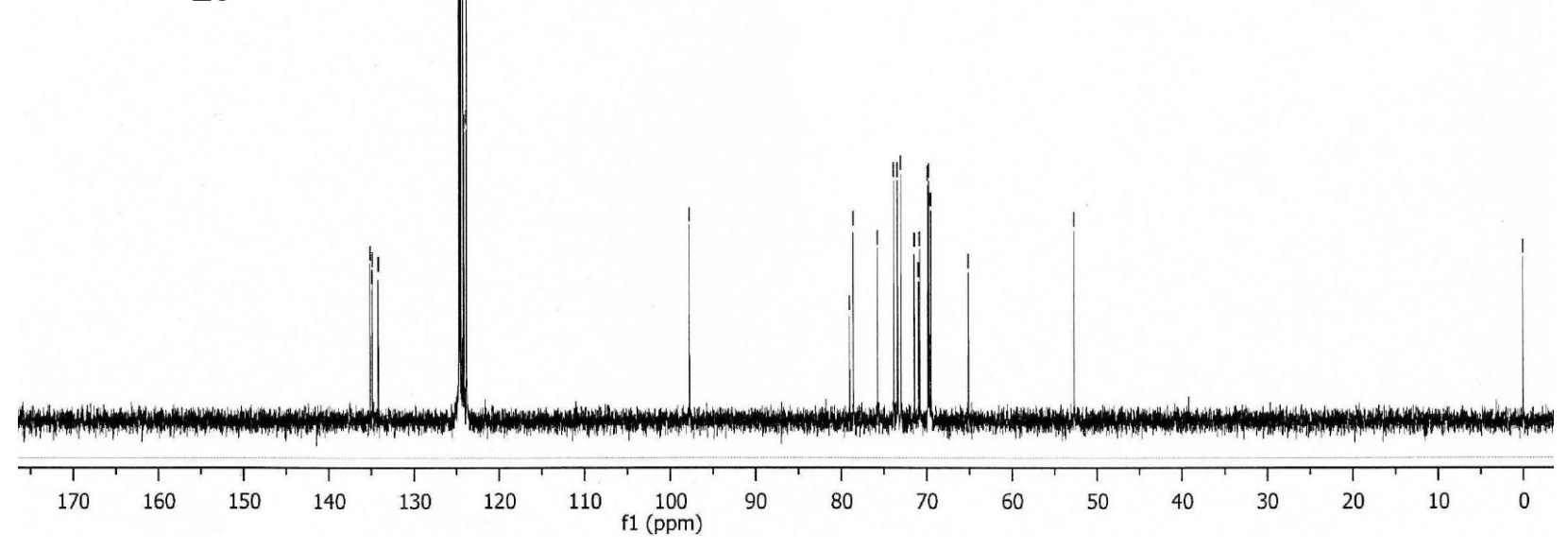




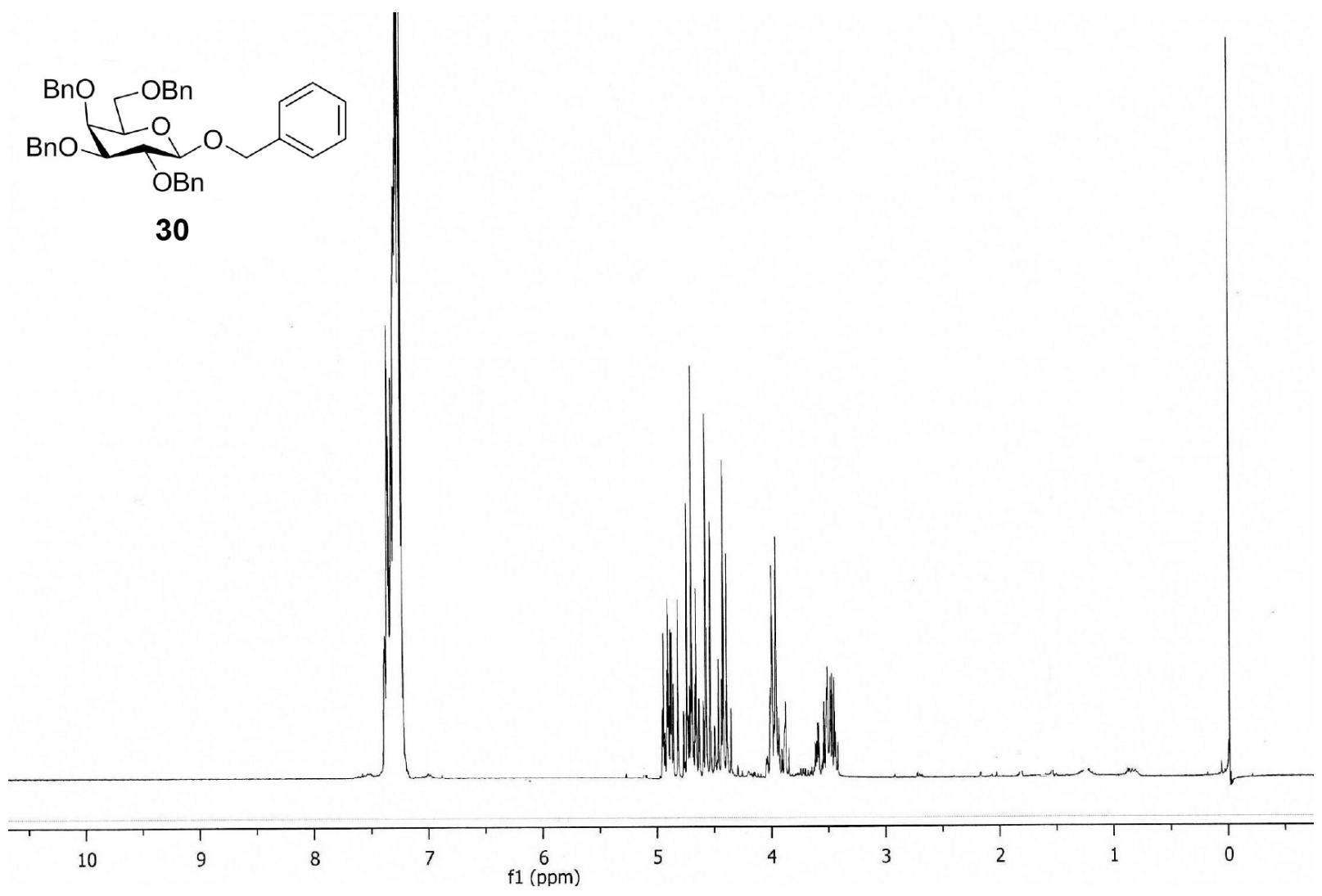

D. Mass Spectral data of the glycosylated products.

\begin{tabular}{|c|c|c|c|}
\hline Entry & $\begin{array}{c}\text { Compound } \\
\text { number }\end{array}$ & $\begin{array}{c}\text { Calculated } \\
\text { Mol. Wt. }\end{array}$ & $\begin{array}{c}\text { Observed } \\
\text { Mol. Wt. }\end{array}$ \\
\hline 1 & $\mathbf{1 0}$ & 220.09 & $243.11(\mathrm{M}+23)$ \\
\hline 2 & $\mathbf{1 1}$ & 270.11 & $293.13(\mathrm{M}+23)$ \\
\hline 3 & $\mathbf{1 2}$ & 292.12 & $315.11(\mathrm{M}+23)$ \\
\hline 4 & $\mathbf{1 3}$ & 422.18 & $445.10(\mathrm{M}+23)$ \\
\hline 5 & $\mathbf{1 4}$ & 262.14 & $285.20(\mathrm{M}+23)$ \\
\hline 6 & $\mathbf{1 5}$ & 220.09 & $243.12(\mathrm{M}+23)$ \\
\hline 7 & $\mathbf{1 6}$ & 270.11 & $293.01(\mathrm{M}+23)$ \\
\hline 8 & $\mathbf{1 7}$ & 292.12 & $315.15(\mathrm{M}+23)$ \\
\hline 9 & $\mathbf{1 8}$ & 422.18 & $445.11(\mathrm{M}+23)$ \\
\hline 10 & $\mathbf{1 9}$ & 262.14 & $285.04(\mathrm{M}+23)$ \\
\hline 11 & $\mathbf{2 0}$ & 220.09 & $243.10(\mathrm{M}+23)$ \\
\hline 12 & $\mathbf{2 1}$ & 270.11 & $293.04(\mathrm{M}+23)$ \\
\hline 13 & $\mathbf{2 3}$ & 422.18 & $445.05(\mathrm{M}+23)$ \\
\hline 15 & $\mathbf{2 4}$ & 262.14 & $285.01(\mathrm{M}+23)$ \\
\hline 16 & $\mathbf{2 6}$ & 584.23 & $607.30(\mathrm{M}+23)$ \\
\hline 17 & $\mathbf{3 1}$ & 630.30 & $653.30(\mathrm{M}+23)$ \\
\hline
\end{tabular}

